\title{
Effect of drying temperature on sensory and flavor of thyme
}

\author{
Chao Zhang ${ }^{\mathrm{a}}$, Jiangchangmei Lu ${ }^{\mathrm{b}}$, Yubin $\mathrm{Wang}^{\mathrm{c}}$, Yue $\mathrm{Ma}^{\mathrm{d}}$, Xiaoyan Zhao ${ }^{\mathrm{e}}$
}

Beijing Vegetable Research Center, Beijing Academy of Agriculture and Forestry Sciences; Beijing

Key Laboratory of Fruits and Vegetable Storage and Processing; Key Laboratory of Biology and Genetic Improvement of Horticultural Crops (North China), Ministry of Agriculture; Key Laboratory of Urban Agriculture (North), Ministry of Agriculture, 9 Shuguanghuayuan Road, Haidian District, Beijing, China

azhangchao@nercv.org, 'blujiangchangmei@nercv.org, 'wangyubin@nercv.org, 'mayue@nercv.org, ezhaoxiaoyan@nercv.org

Keywords: thyme, drying, flavor, sensory, greenness

Abstract. The effect of drying temperature on sensory and flavor of thyme was evaluated. The drying temperature showed no influence on the flavor the dehydrated thyme. A higher drying temperature leaded to a dark greenness of the thyme. Hence, a higher drying temperature leaded to an attractive appearance of the dehydrated thyme.

\section{Introduction}

Thyme (Thymus vulgaris L.), a plant species native to the western Mediterranean area, is now widely cultivated throughout temperate climes [1]. The thyme has been popularly used as a culinary herb for flavouring and seasoning [2].

Volatile aroma compounds are the most sensitive components in the process of food drying. The effect of drying on the composition of volatile flavor constituents of various aromatic plants and vegetables has been the subject of numerous studies $[3,4]$, which show that the changes in the concentrations of the volatile compounds during drying depend on several factors, such as the drying method and parameters that are characteristic of the product subjected to drying [5].

Thyme is usually dehydrated in an air-circulated over [6, 7]. However, the sensory and flavor of the dehydrated thyme was neglected to our knowledge. The drying temperature was an important factor influencing the final quality of the products. Hence, the effect of drying temperature on sensory and flavor of thyme was evaluated. The flavor and appearance of the dehydrated thyme was compared.

\section{Material and Methods}

Dehydration of thyme. Thyme was picked from our Tongzhou farm (Tongzhou District Beijing, 2014). The fresh leaf of the thyme was stored at $4{ }^{\circ} \mathrm{C}$ before use. The thyme was washed by the tap water at $4{ }^{\circ} \mathrm{C}$ to remove the soil and some inclusion. The washed thyme was heated at 55, 65, 75, 85 and $95{ }^{\circ} \mathrm{C}$ in a air-circulated oven respectively. The moisture content of $7 \%$ was the termination for each treatment. During each treatment, the products were sampled for 7 9 times for the quality determination. The fresh thyme was designated as the control.

Color determination. The sample was powdered and measured by a reflective mode in a $0.5 \mathrm{~cm}$ cuvette, followed the recently reported method[8]. The color of samples was assessed in a LAB space with the dimension $\boldsymbol{L}^{*}$ for lightness and $\boldsymbol{a}^{*}$ and $\boldsymbol{b}^{*}$ for the color-opponent dimensions by a spectrophotometer (CM3700d, Konica Minolta Sensing INC., Japan). Specifically, the value $L^{*}$ 
represents the lightness of the color $\left(\boldsymbol{L}^{*}=0\right.$ yields black and $\boldsymbol{L}^{*}=100$ indicates diffuse white); the negative value $\boldsymbol{a}^{*}$ indicates green while positive values indicate magenta; the negative value $\boldsymbol{b}^{*}$ indicates blue and positive values indicate yellow. The fresh thyme was measured as the control.

Flavor comparison. The flavor of the samples was compared by an electronic nose PEN2 (Airsense Analytics GmbH, Schwerin, Germany), followed the recently method [9]. The electronic nose was turned on for $30 \mathrm{~min}$ and flushed the testing system for $180 \mathrm{~s}$. The sample of $2 \mathrm{ml}$ was put in the testing tube. And then the electronic sensor was put into the testing tube to collect the results for $60 \mathrm{~s}$. The response of the sensor in 48 52 s were evaluated by a principal component analysis. The fresh thyme was designated as the control.

Statistical Analysis. Analysis of variance (ANOVA) was used to compare mean differences of the results. If the differences in mean existed, multiple comparisons were performed using Duncan's Multiple Range Test. All analysis was conducted using SPSS for Window Version 19. All experiments were done in triplicates or more.

\section{Results and Discussion}

Effect of drying temperature on color of thyme. The effect of drying temperature on color of thyme is shown in Figure 1. The color of the samples was expressed by the LAB system. the value $\boldsymbol{L}^{*}$ represents the lightness of the color $\left(\boldsymbol{L}^{*}=0\right.$ yields black and $\boldsymbol{L}^{*}=100$ indicates diffuse white) $[10,11]$. The $\boldsymbol{L}^{*}$ of the samples was enhanced significantly after drying at each temperature. The $\boldsymbol{L}^{*}$ of the samples dried at 75 , and $85^{\circ} \mathrm{C}$ was significant higher than that dried at 45,55 and $65^{\circ} \mathrm{C}$.

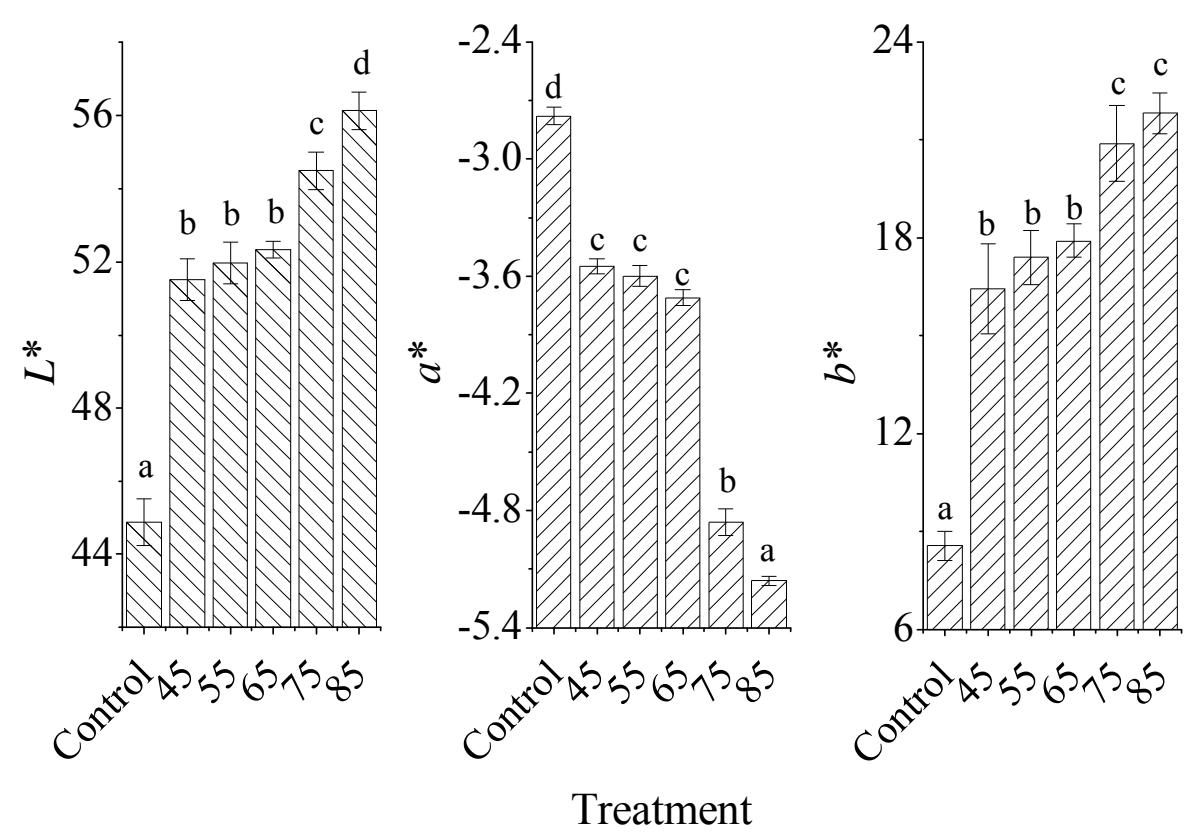

Figure 1 Effect of drying temperature on color of dehydrated thyme

The negative value $a^{*}$ indicates green while positive values indicate magenta [12]. The $a^{*}$ value is closely related to the greenness of a sample. The greenness of the thyme was mainly came from the chlorophyll. Consequently, the $a^{*}$ value was an indicator of the chlorophyll content. The chlorophyll content is decrease due to its degradation. The chlorophyll degradation of the spinach [13] and kiwifruit puree [14] is found to follow the first-order kinetics. The $a^{*}$ of the thyme dried at 75 and $85{ }^{\circ} \mathrm{C}$ was significant lower than that dried at 45,55 and $65{ }^{\circ} \mathrm{C}$. Consequently, the greenness of the thyme dried at 45,55 and $65{ }^{\circ} \mathrm{C}$ was lighter than that dried at the other temperature. The phenomenon proved that a higher temperature benefitted to hold the original color of the dehydrated thyme. 
The negative value $\boldsymbol{b}^{*}$ indicates blue and positive values indicate yellow [12]. The $b^{*}$ value was increased when the drying temperature was enhanced. The $b^{*}$ of the thyme dried at 75 and $85{ }^{\circ} \mathrm{C}$ was significant higher than dried at the other temperature.

Effect of drying temperature on flavor of thyme. The effect of drying temperature on flavor of thyme is shown in Figure 2. The principal component analysis showed that the flavor of the samples was mainly contributed by the main component 1 and main component 2 . The main component 1 and main component 2 contributed $99.92 \%$ and $0.03 \%$ for the flavor of the thyme. The main component 1 and 2 accounted $99.95 \%$ of the total flavor, which was effective to reflect the flavor of the thyme. Each drying temperature leaded to a significant difference compared with the flavor of the control. Remarkably, the flavor of each thyme was similar. Consequently, the drying temperature showed no influence on the flavor of the dehydrated thyme. Being different to our results, the flavor of spearmint and peppermint is affected be the drying temperature [3, 4].

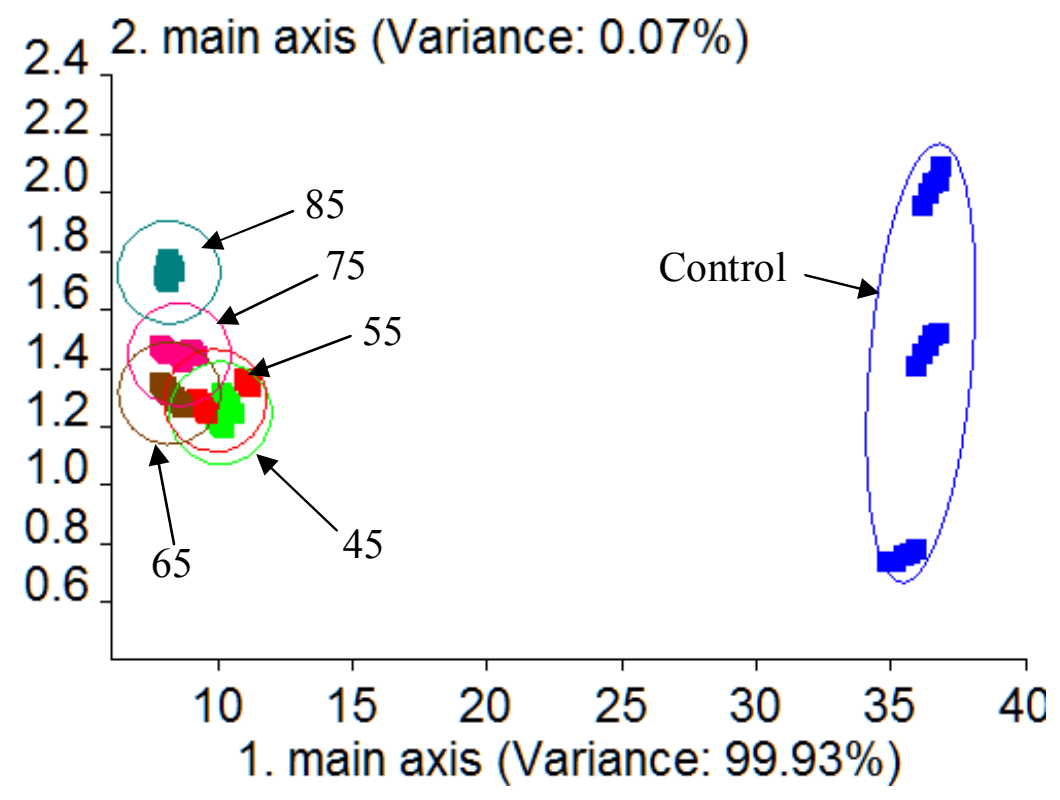

Figure 2 Effect of drying temperature on flavor of dehydrated thyme

Effect of dehydration on appearance of thyme. The effect of drying temperature on appearance of thyme is shown in Figure 3. The color of the dehydrated thyme was a little yellow to the color of the fresh thyme. Moreover, the drying temperature also affected the greenness and yellowness of the thyme. The thyme dehydrated at $85{ }^{\circ} \mathrm{C}$ showed dark green, while that dehydrated at $45{ }^{\circ} \mathrm{C}$ showed light green. The phenomenon was also been validated by the color evaluation. On the other hand, a higher temperature leaded to a browning of the product [15]. The Consequently, the appearance of the thyme was the summary of greenness and browning. The accumulated green and browning leaded to the dark green. Consumers would prefer the dark green, due to the greenness of the fresh thyme. Hence, the a higher drying temperature was preferred from the appearance of the dehydrated thyme. 

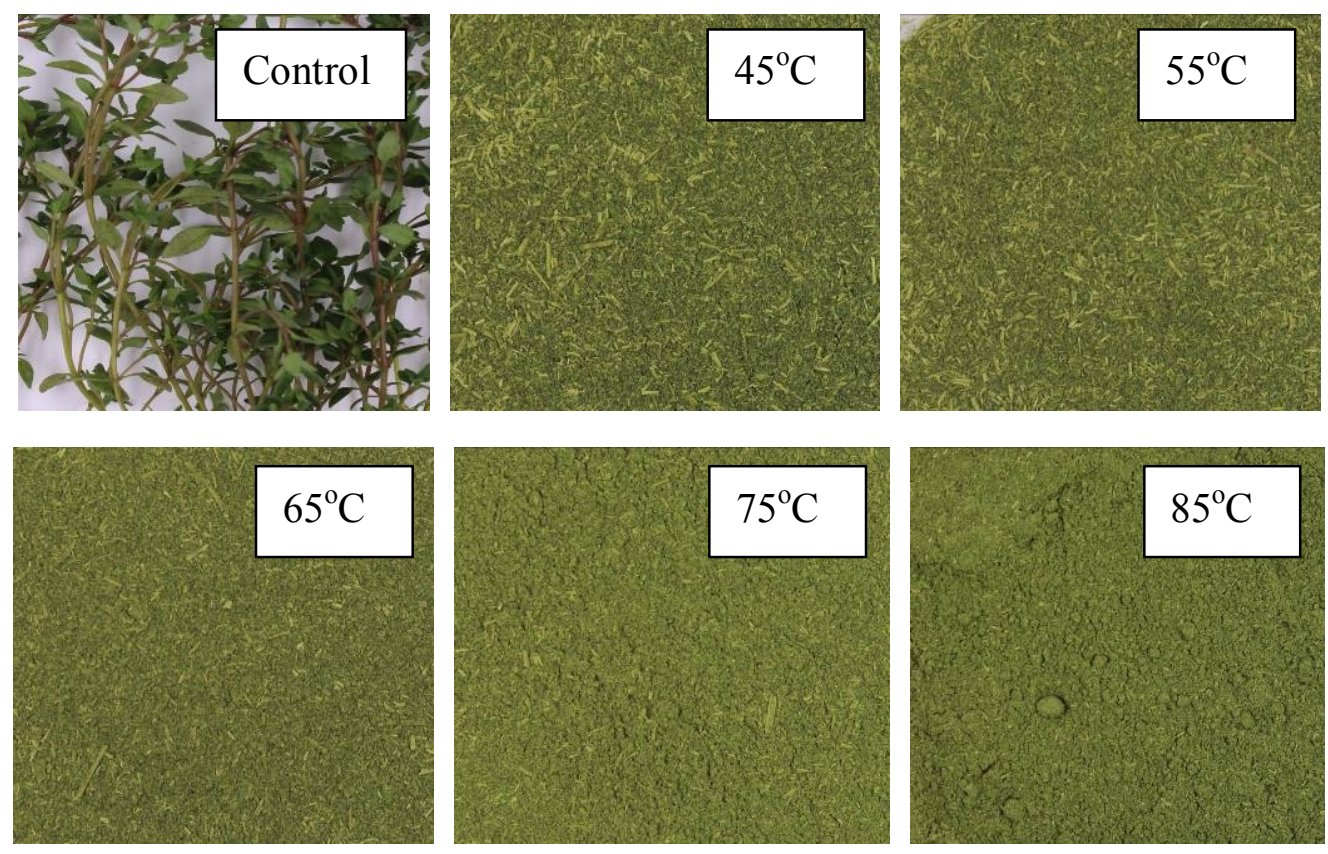

Figure 3 Effect of drying temperature on appearance of dehydrated thyme

\section{Conclusions}

The drying temperature showed no influence on the flavor the dehydrated thyme. A higher drying temperature leaded to a dark greenness of the thyme. Hence, a higher drying temperature would benefited the appearance of the dehydrated thyme.

\section{Acknowledgements}

The authors are grateful to financial support of the earmarked fund for Modern Agro-industry Technology Research System (CARS-26-22 \& CARS-25), Beijing Academy of Agricultural and Forestry Sciences, New Discipline Breeding (KJCX20140204), and Beijing Key Laboratory of Fruits and Vegetable Storage and Processing (Z141105004414037).

\section{References}

[1] Z. Alcicek: Food Chem. Vol 128 (2011) 683.

[2] S. Kykkidou, V. Giatrakou, A. Papavergou, M. G. Kontominas, I. N. Savvaidis: Food Chem. Vol 115 (2009) 169.

[3] R. Baranauskienė, E. Bylaitė, J. Žukauskaitè, P. R. Venskutonis: J. Agric. Food Chem. Vol 55 (2007) 3027.

[4] M. C. Díaz-Maroto, M. S. Pérez-Coello, M. A. G. Viñas, M. D. Cabezudo: J. Agric. Food Chem. Vol 51 (2003) 1265.

[5] P. R. Venskutonis: Food Chem. Vol 59 (1997) 219.

[6] J. Wu, C. Yang, Y. Rong, Z. Wang: Procedia Eng. Vol 37202.

[7] T. Nakatsu, A. T. Lupo Jr, J. W. Chinn Jr, R. K. L. Kang, R. Atta ur, Elsevier, 2000, 571.

[8] J. Tian, X. Zeng, S. Zhang, Y. Wang, P. Zhang, A. Lu, X. Peng: Indust. Crops Prod. Vol 59 (2014) 69.

[9] M. Laureati, S. Buratti, A. Bassoli, G. Borgonovo, E. Pagliarini: Food Res. Int. Vol 43 (2010) 959.

[10] S. A. Osmani, E. H. Hansen, C. Malien-Aubert, C. E. Olsen, S. Bak, B. L. Mller: J. Agric. 
Food Chem. Vol 57 (2009) 3149.

[11] T. P. Labuza: J. Food Sci. Vol 51 (1986) ii.

[12] L. Rolle, S. Guidoni: J. Int. Des Sciences De La Vigne Et Du Vin Vol 41 (2007) 193.

[13] F. M. Lajolo, U. M. Lanfer Marquez: J. Food Sci. Vol 47 (1982) 1995.

[14] M. Benlloch-Tinoco, A. Kaulmann, J. Corte-Real, D. Rodrigo, N. Martinez-Navarrete, T. Bohn: Food Chem. Vol 187 (2012) 254.

[15] T. Imaizumi, T. Orikasa, S. Morifusa, L. V. Man, Y. Muramatsu, S. Koide, T. Uchino, F. Tanaka, D. Hamanaka, A. Tagawa: Eng. Agric., Environm. Food Vol 8 (2015) 1. 